\title{
BMJ Open Associations between regular GP contact, diabetes monitoring and glucose control: an observational study using general practice data
}

\author{
David Youens (D) , ${ }^{1}$ Suzanne Robinson (D) , ${ }^{1}$ Jenny Doust, ${ }^{2}$ Mark N Harris, ${ }^{3}$ \\ Rachael Moorin (i) 1,4
}

To cite: Youens D, Robinson S, Doust J, et al. Associations between regular GP contact, diabetes monitoring and glucose control: an observational study using general practice data. BMJ Open 2021;11:e051796. doi:10.1136/ bmjopen-2021-051796

\section{- Prepublication history and} additional supplemental material for this paper are available online. To view these files, please visit the journal online (http://dx.doi.org/10.1136/ bmjopen-2021-051796).

Received 29 March 2021 Accepted 13 October 2021

\section{Check for updates}

(C) Author(s) (or their employer(s)) 2021. Re-use permitted under CC BY-NC. No commercial re-use. See rights and permissions. Published by BMJ.

${ }^{1}$ School of Population Health, Curtin University, Bentley, Western Australia, Australia ${ }^{2}$ Centre for Longitudinal and Life Course Research, School of Public Health, The University of Queensland, Brisbane, Queensland, Australia

${ }^{3}$ School of Accounting, Economics \& Finance, Curtin University, Bentley, Western Australia, Australia

${ }^{4}$ School of Population \& Global Health, University of Western Australia, Perth, Western Australia, Australia

Correspondence to

David Youens;

david.youens@curtin.edu.au

\section{ABSTRACT}

Objective Continuity and regularity of general practitioner (GP) contacts are associated with reduced hospitalisation in type 2 diabetes (T2DM). We assessed associations of these GP contact patterns with intermediate outcomes reflecting patient monitoring and health.

Design Observational longitudinal cohort study using general practice data 2011-2017.

Setting 193 Australian general practices in Western Australia and New South Wales participating in the Medicinelnsight programme run by NPS MedicineWise. Participants 22791 patients aged 18 and above with T2DM.

Interventions Regularity was assessed based on variation in the number of days between GP visits, with more regular contacts assumed to indicate planned, proactive care. Informational continuity (claims for care planning incentives) and relational continuity (usual provider of care index) were assessed separately.

Outcome measures Process of care indicators were glycosylated haemoglobin ( $\mathrm{HbA1c}$ ) test underuse (8 months without test), estimated glomerular filtration rate (eGFR) underuse (14 months) and $\mathrm{HbA1c}$ overuse (two tests within 80 days). The clinical indicator was T2DM control (HbA1c 6.5\% (47.5 mmol/mol)-7.5\% (58.5 mmol/ mol)).

Results The quintile with most regular contact had reduced odds of $\mathrm{HbA} 1 \mathrm{C}$ and eGFR underuse (OR 0.74 , $95 \% \mathrm{Cl} 0.67$ to 0.81 and $\mathrm{OR} 0.78,95 \% \mathrm{Cl} 0.70$ to 0.86 respectively), but increased odds of $\mathrm{HbA1c}$ overuse (OR $1.20,95 \% \mathrm{Cl} 1.05$ to 1.38). Informational continuity was associated with reduced odds of $\mathrm{HbA} 1 \mathrm{c}$ underuse $(\mathrm{OR}$ $0.53,95 \% \mathrm{Cl} 0.49$ to 0.56$)$, reduced eGFR underuse (OR $0.62,95 \% \mathrm{Cl} 0.58$ to 0.67$)$ and higher odds of $\mathrm{HbA} 1 \mathrm{C}$ overuse (OR 1.48, 95\% Cl 1.34 to 1.64). Neither had significant associations with $\mathrm{HbA} 1 \mathrm{c}$ level. Results for relational continuity differed.

Conclusions This study provides evidence that regularity and continuity influence processes of care in the management of patients with diabetes, though this did not result in the recording of $\mathrm{HbA} 1 \mathrm{c}$ within target range. Research should capture these intermediate outcomes to better understand how GP contact patterns may influence health rather than solely assessing associations with hospitalisation outcomes.

\section{STRENGTHS AND LIMITATIONS OF THIS STUDY}

$\Rightarrow$ The general practice data used allowed for assessment of both processes of care and clinical outcomes.

$\Rightarrow$ The general practice data used provided for a large cohort and allowed for control of a range of important covariates.

$\Rightarrow$ Information on practices not participating in the Medicinelnsight programme was unavailable, impacting interpretation.

$\Rightarrow$ The data collection used did not capture hospital use, preventing a mediation analysis capturing hospitalisation outcomes.

\section{INTRODUCTION}

Continuity of care refers to a relationship between a practitioner and patient extending beyond a single episode of illness, implying some affiliation between patient and provider. ${ }^{1}$ Continuity is typically measured by assessing whether a patient has repeated contact with one provider. Our group has assessed this on the basis of the regularity of general practitioner (GP) contact over time, with visits on a regular basis taken to indicate planned, proactive care. Continuity and regularity of GP contact are associated with hospitalisation outcomes among patients with diabetes. ${ }^{2-6}$ Regularity and continuity are assumed to prevent hospitalisation and mortality via intermediate outcomes such as improved monitoring of the patient by the $\mathrm{GP},{ }^{7}$ facilitating detection and responses to deteriorations in condition, ${ }^{4}$ improved medical management and improved patient compliance. ${ }^{9}$ Associations reported between these exposures and hospitalisation outcomes may be subject to unobserved confounding, as this research is typically observational. ${ }^{10}$ Given the hypothesised mechanisms of action via intermediate outcomes, evidence concerning associations 
with these intermediate outcomes is valuable. Intermediate outcomes are categorised here as (1) processes of care, that is, improved patient monitoring or appropriate prescribing and (2) clinical outcomes, that is, objective measures of patient health. ${ }^{11}$ It has previously been demonstrated that in patients at risk of cardiovascular disease events, regular care and higher continuity were associated with improved statin use, providing some evidence regarding processes of care. ${ }^{12}$

As this work is conducted in Australia, a description of the role of the GP in this setting is warranted. GPs have major responsibility for prescribing and ordering of diagnostic tests; over $60 \%$ of encounters result in a prescription and about one-quarter result in diagnostic investigation. ${ }^{13}$ In Australia GPs have a role as gatekeepers, with specialist care requiring a GP referral, ${ }^{14}$ and referrals to allied health and hospital also common. ${ }^{13}$ GPs operate on a fee-for-service basis with the Federal Government reimbursing GPs for each encounter via Medicare, Australia's universal public insurance programme, though providers may charge additional copayments. ${ }^{15}$ Practices are private business, made up of one or more GPs operating as a business unit. A point relevant to continuity is that patients do not formally register with a specific GP or practice, instead people can switch providers at any time. ${ }^{14}$ Meanwhile, there is a trend towards larger practice sizes. ${ }^{13}$

Much research in this area has relied on the use of administrative (financial) data collections. These provide comprehensive information on services rendered (eg, GP contacts, medication dispensations, hospitalisations) but often lack detailed clinical information. ${ }^{16}$ Recently the information captured in general practice clinical information systems has started becoming available to researchers. These provide a potentially rich source of data, containing more detailed clinical information than administrative collections. ${ }^{17}$ Importantly for the current work, these include pathology test results. Pathology test results are not generally available in administrative collections and in Australia, administrative data often do not clearly indicate the completion of specific pathology tests as national reimbursement databases often use a single code for multiple tests attracting the same reimbursement. ${ }^{17}$

The aim of the current work was to estimate the impact of GP regularity and continuity on (1) diabetes processes of care, as indicated by the completion of pathology tests according to clinical guidelines and (2) clinical outcomes, as indicated by glycosylated haemoglobin ( $\mathrm{HbA1c}$ ) values. This work focusses on type 2 diabetes mellitus (T2DM) due to its high prevalence, ${ }^{18}$ the availability of comprehensive management guidelines ${ }^{19}$ and the central role of the GP in its management. ${ }^{20}$ Clinical guidelines are useful in such research as they outline clear regimens for prescribing and pathology testing, and targets on clinical indicators. ${ }^{19}$ These guidelines do not intend to provide a prescriptive approach for all patients with a condition, considering the heterogeneous populations clinicians manage, but at the population level researchers can use the recommendations in guidelines to compare management of conditions across different groups.

Studies have assessed the impact of sex and socioeconomic status on similar outcomes in cohorts with chronic heart disease. ${ }^{21} 22$ These found that both male gender and lower socioeconomic status were associated with improved care processes (prescribing and completion of monitoring tests), but worse clinical outcomes (based on pathology test results). This highlights the importance of assessing both sets of outcomes to improve the understanding of potential impacts of any explanatory variables on patient management and health.

\section{METHODS}

This was an observational longitudinal cohort study consisting of a secondary analysis of longitudinal general practice data. Reporting follows the REporting of studies Conducted using Observational Routinely-collected Data (RECORD) statement. ${ }^{23}$

\section{Data}

Data were a collection of person-level deidentified general practice clinical information system data, called MedicineInsight, collated by NPS MedicineWise, which is a non-profit organisation aiming to promote the quality use of medicines. ${ }^{24}$ MedicineInsight was established in 2011 with funding by the Australian Government, with the primary aim of supporting postmarket medication surveillance. ${ }^{24}$ General practices are recruited to MedicineInsight on a practice opt-in, patient opt-out basis, meaning that when a practice opts in data on all GPs and all patients (except those who explicitly opt-out) will become available. Following recruitment, historical patient data are extracted, deidentified, encrypted and transmitted to the primary care database with ongoing monthly data extracts. Data include patient demographics, diagnoses, prescriptions, pathology results and immunisations, and more. ${ }^{17}$ As of October 2018, MedicineInsight had recruited 662 practices across Australia (8.2\% of all practices).$^{24}$ This study used data from all participating practices in Western Australia $(\mathrm{N}=53)$ and New South Wales $(\mathrm{N}=140)$ from the start of the collection up to late 2017. Compared with the general population visiting general practices in Australia, patients captured in MedicineInsight data are slightly more likely to be female ( $55.7 \%$ vs $52.4 \%$ ), are similarly likely to be Indigenous $(2.6 \%$ vs $2.9 \%)$, have a similar distribution of age and socioeconomic status, though some states are over-represented compared with others. ${ }^{25}$ In some cases, multiple physical practices share a Clinical Information System (CIS), and in these cases the multiple practices are considered a single 'site' within the database regardless of the number of physical practices. Approximately $90 \%$ of sites are composed of a single practice. ${ }^{24}$ As part of their participation in the programme, practices receive information on their own care delivery and prescribing, including benchmarking against other providers and 
practices, so as to support quality improvement in primary care. ${ }^{26}$ MedicineInsight data can be used for beneficial research following approval by the Independent Data Governance Committee. $^{27}$

\section{Design}

The data were organised into a longitudinal design to reduce risk of reverse causation. A pre-exposure period ran from 1 January 2011 to 28 February 2015, during which the cohort was identified and baseline patient characteristics assessed; an exposure period from 1 March 2015 to 29 February 2016, during which exposure variables were ascertained; and outcomes assessed during a follow-up period from 1 March 2016 to 30 June 2017. A diagram is provided in online supplemental file 1.

\section{Cohort}

A cohort with T2DM was captured based on recorded diagnoses and the reasons for prescription with each prescription record. These fields were text strings and included records generated by clinical information systems (ie, drop down menus) and records entered manually by GPs, hence there was potential for typing errors and a need to manually verify diagnosis data. As there were millions of records a procedure to expedite manual review was used, explained in online supplemental file 2.

Those over 80 were excluded, since HbAlc goals differ for older patients with higher risk of hypoglycaemia. ${ }^{28}$

\section{Exposures}

Regularity of GP contact was based on all contacts with GPs during the exposure period, and refers to the distribution of these contacts over time. Regularity was calculated using a previously described index, based on the variation in the number of days between GP contacts. ${ }^{3}$ In brief, for each GP contact the number of days since the prior contact was counted, and the coefficient of variation in this number of days calculated. An index $(\mathrm{R})$ was calculated by $\mathrm{R}=1 / 1+$ (coefficient of variation (days between visits)). This ranges from 0 (least regular) to 1 (most regular). For example, if a patient had four GP contacts within a year, three of which were in January and one in December, they would have a lower regularity score than a patient with four visits in January, March, August and December. This index was split into quintiles based on the distribution within the cohort.

Two exposures assessed continuity of care. The first indicated whether a GP had claimed one of a set of chronic disease management (CDM) financial incentives reimbursed via Medicare during the exposure period including preparation or review of a GP management plan, co-ordination of team care arrangements or contribution to a multidisciplinary care plan. ${ }^{29}$ Claiming these items in relation to a patient is taken to indicate informational continuity (ie, information on past events and circumstances is available so appropriate care can be provided, ${ }^{1}$ following Ride $e t a l^{10}$
The Usual Provider of Care (UPC) index was also used. This captures the proportion of all GP visits made to the usual provider during the exposure period ${ }^{30}$ (ie, the provider the patient visited most often). For example, if a patient visits provider A three times during the exposure period and provider B once, their UPC score will be 0.75 . This is a measure of interpersonal continuity (an ongoing relationship between the patient and provider $)^{1}$ UPC was categorised as low $(0-0.39)$, intermediate $(0.4-$ $0.59)$, high (0.6-0.99) and perfect (UPC $=1)$. Though this is usually categorised, cut-off values differ ${ }^{67}$ with no gold standard. This index is included as visit-based measures are the most commonly used continuity measures. In the current data patient identifiers are practice-specific, meaning that a patient visiting two separate practices will appear as two separate patients, while visits to practices not participating in MedicineInsight are not captured. If a patient visits multiple practices, of which only one participates in MedicineInsight, they may record a high UPC despite having lower continuity in reality. This is referred to as 'practice-specific UPC'.

The cohort was restricted to patients with at least three GP contacts as the regularity score cannot be calculated with fewer than three contacts.

\section{Outcomes}

Outcomes were processes of care and clinical indicators developed using pathology testing records. These records were electronically coded in contrast to the freetext diagnosis fields described earlier. We assessed indicators concerning management of HbAlc, as one of the most important markers of diabetes control, and the estimated glomerular filtration rate (eGFR), as a marker of the development of one of the most important diabetes complications.

Processes of care relate to pathology testing within timeframes recommended by guidelines. ${ }^{19}$ These recommend HbAlc testing every 6 months, with a maximum frequency of every 3 months. Guidelines also recommend testing the eGFR every twelve months (along with other tests not assessed here). Three process of care outcomes were defined. The first two were underuse of HbAlc, defined as any 8-month period during follow-up without an HbAlc test; and underuse of eGFR, defined as any 14-month period during the follow-up without a test. Underuse represents potentially inappropriate care as changes in health and opportunities to adjust treatment may be missed. The third was overuse of HbAlc, defined as two tests within 80 days. Overuse may be problematic as HbAlc reflects glycaemic control over 3 months (the halflife of red blood cells) ${ }^{31}$ hence tests within this window are not informative. More frequent use represents wasted resources $^{32}$ and may impact clinical decision making. For example, where medications are changed following a test indicating poor glycaemic control, a follow-up test within 3 months may incorrectly suggest that the treatment has not worked as the effects of the medication change will not yet be fully evident, potentially leading 
to further unnecessary medication changes and risk of hypoglycaemia. ${ }^{32}$

The clinical indicator was an $\mathrm{HbAlc}$ result within treatment targets. Participants were flagged as being outside or within target range $(6.5 \%(47.5 \mathrm{mmol} / \mathrm{mol})-7.5 \%$ $(58.5 \mathrm{mmol} / \mathrm{mol}))$ on the first test during the follow-up. ${ }^{19}$ The cohort here differed, as those without an HbA1c test during follow-up were excluded. Treatment targets may be modified for some patients based on clinical judgement. Patient-specific treatment targets are not available in the data, however, and at the cohort level an overall indicator was considered appropriate as adjustments to targets for some patients are unlikely to systematically bias analyses. A variable indicating an HbAlc result within target on any test during the follow-up was not considered as this would depend on both clinical outcomes and processes of care (number of tests).

\section{Covariates}

Patient characteristics included sex, age, rurality (Accessibility and Remoteness Index of Australia (ARIA) ), ${ }^{33}$ state of residence, Indigenous and smoking status. Baseline HbAlc was based on the final test in the pre-exposure period. A measure of comorbidity was calculated based on the RxRisk comorbidity index. ${ }^{34}$ The RxRisk index identifies up to 46 conditions, based on an individual having prescriptions for medications pathognomic for these conditions. The index provides an integer ranging from 0 to 46 indicating the number of RxRisk conditions present, based on 5 years of prescription data prior to the exposure period. The frequency of GP contacts in the exposure period was also captured, that is, the count of visits made by the patient to the general practice captured in the CIS. Categories for all covariates are displayed in table 1. Missing values were treated as a separate category so as to prevent data loss in regression models.

Practice-level characteristics included the SocioEconomic Index for Areas-Index of Relative Social Disadvantage decile of the practice ${ }^{35}$ and practice rurality (ARIA). Practice size was based on the number of GPs working at each practice.

\section{Statistical analysis}

Descriptive statistics were generated for sociodemographics, health service use variables, outcomes and practice characteristics.

Data were multilevel, with patients nested within practices. Patients may see multiple GPs at one practice hence patients were not nested within providers. Patients may also visit multiple practices, but in the data patient IDs were practice-specific, hence patients could not be tracked across practices. Random intercept logistic regression models were used, common in analysing general practice data. ${ }^{36}$ The same analysis was applied to all outcomes. Intraclass correlation coefficients (ICCs) were calculated to determine the proportion of variation in outcomes determined by the practice level by fitting
Table 1 Characteristics of cohort and practices contributing data

\begin{tabular}{|c|c|c|c|}
\hline Variable & & $\mathbf{N}$ & $\%$ \\
\hline \multicolumn{4}{|c|}{ Sociodemographics } \\
\hline \multirow[t]{3}{*}{ Sex } & Male & 12349 & 54.18 \\
\hline & Female & 10437 & 45.79 \\
\hline & Not stated & $<5$ & - \\
\hline \multirow[t]{6}{*}{ Age } & 20-29 & 167 & 0.73 \\
\hline & 30-39 & 826 & 3.62 \\
\hline & $40-49$ & 2301 & 10.10 \\
\hline & $50-59$ & 4951 & 21.72 \\
\hline & $60-69$ & 7792 & 34.19 \\
\hline & $70-79$ & 6754 & 29.63 \\
\hline \multirow[t]{6}{*}{ Rurality } & Major cities & 14391 & 63.14 \\
\hline & Inner regional & 5797 & 25.44 \\
\hline & Outer regional & 2216 & 9.72 \\
\hline & Remote & 256 & 1.12 \\
\hline & Very remote & 50 & 0.22 \\
\hline & Missing & 81 & 0.36 \\
\hline \multirow[t]{2}{*}{ State } & New South Wales & 15847 & 69.53 \\
\hline & Western Australia & 6944 & 30.47 \\
\hline \multirow[t]{3}{*}{ Indigenous status } & Aboriginal/TSI & 865 & 3.80 \\
\hline & Neither & 18781 & 82.41 \\
\hline & Not stated & 3145 & 13.80 \\
\hline \multirow[t]{4}{*}{ Smoking } & Smoker & 2826 & 12.40 \\
\hline & Ex-smoker & 8230 & 36.11 \\
\hline & Non-smoker & 10652 & 46.74 \\
\hline & Not stated & 1083 & 4.75 \\
\hline
\end{tabular}

Health service use (exposure period) ${ }^{\star}$

\begin{tabular}{|c|c|c|c|}
\hline \multirow[t]{4}{*}{ Frequency } & $0-4$ & 8276 & 36.31 \\
\hline & 5-9 & 8161 & 35.81 \\
\hline & $10-14$ & 3559 & 15.62 \\
\hline & $15+$ & 2795 & 12.26 \\
\hline \multirow[t]{4}{*}{ Practice-specific UPC } & $0-0.39$ & 6657 & 29.21 \\
\hline & $0.4-0.59$ & 5231 & 22.95 \\
\hline & $0.6-0.99$ & 3507 & 15.39 \\
\hline & & 7396 & 32.45 \\
\hline \multirow[t]{2}{*}{ CDM† item } & No & 8125 & 35.65 \\
\hline & Yes & 14666 & 64.35 \\
\hline \multirow{5}{*}{$\begin{array}{l}\text { Rx-risk comorbidity } \\
\text { conditions } \ddagger\end{array}$} & 0 & 874 & 3.83 \\
\hline & $1-2$ & 3852 & 16.90 \\
\hline & $3-4$ & 5577 & 24.47 \\
\hline & $5-6$ & 5339 & 23.43 \\
\hline & $7+$ & 7149 & 31.37 \\
\hline \multicolumn{4}{|c|}{ Outcome variables (outcome period)§ } \\
\hline \multirow[t]{2}{*}{ HbA1c overuse } & No & 19525 & 85.67 \\
\hline & Yes & 3266 & 14.33 \\
\hline \multirow[t]{2}{*}{ HbA1c underuse } & No & 9129 & 40.06 \\
\hline & Yes & 13662 & 59.97 \\
\hline
\end{tabular}


Table 1 Continued

\begin{tabular}{|c|c|c|c|}
\hline Variable & & $\mathbf{N}$ & $\%$ \\
\hline \multirow[t]{2}{*}{ eGFR underuse } & No & 16719 & 73.36 \\
\hline & Yes & 6072 & 26.64 \\
\hline \multirow{3}{*}{$\begin{array}{l}\text { HbA1c within target } \\
\text { on first test in follow- } \\
\text { up }\end{array}$} & No & 12708 & 55.76 \\
\hline & Yes & 5667 & 24.87 \\
\hline & No test & 4416 & 19.38 \\
\hline Total & & 22791 & 100 \\
\hline \multicolumn{4}{|l|}{ Practice characteristics } \\
\hline \multirow[t]{2}{*}{ State } & New South Wales & 140 & 72.54 \\
\hline & Western Australia & 53 & 27.46 \\
\hline \multirow[t]{11}{*}{ SEIFA decile } & $\begin{array}{l}1 \text { (most } \\
\text { disadvantage) }\end{array}$ & 9 & 4.66 \\
\hline & 2 & 18 & 9.38 \\
\hline & 3 & 22 & 11.46 \\
\hline & 4 & 24 & 12.50 \\
\hline & 5 & 14 & 7.29 \\
\hline & 6 & 34 & 17.71 \\
\hline & 7 & $\S$ & 4.17 \\
\hline & 8 & 13 & 6.74 \\
\hline & 9 & 26 & 13.54 \\
\hline & $\begin{array}{l}10 \text { (least } \\
\text { disadvantage) }\end{array}$ & 26 & 13.54 \\
\hline & Missing & $<5$ & - \\
\hline \multirow[t]{4}{*}{ Practice rurality } & Major cities & 125 & 64.77 \\
\hline & Inner regional & 39 & 20.21 \\
\hline & Outer regional & 23 & 11.92 \\
\hline & $\begin{array}{l}\text { Remote/very } \\
\text { remote }\end{array}$ & * & 3.11 \\
\hline \multirow[t]{4}{*}{ No of GPs } & 1 & 68 & 35.23 \\
\hline & $2-9$ & 63 & 32.64 \\
\hline & $10-19$ & 45 & 23.32 \\
\hline & $20+$ & 17 & 8.81 \\
\hline \multirow[t]{5}{*}{ No of patients } & $<750$ & 38 & 19.69 \\
\hline & 750-999 & 20 & 10.36 \\
\hline & $1000-1499$ & 54 & 27.98 \\
\hline & 1500-1999 & 29 & 15.03 \\
\hline & $2000+$ & 52 & 26.94 \\
\hline Total & & 193 & 100 \\
\hline
\end{tabular}

*March 2015-February 2016.

†Reimbursement to GP for certain care coordination activities. $\neq$ Over 5-year period to end of exposure.

§March 2016-June 2017.

IWhere cell is $<5$, other values on variable altered to protect confidentiality.

CDM, Chronic Disease Management; eGFR, estimated glomerular filtration rate; GP, general practitioner; HbA1c, glycosylated haemoglobin; SEIFA, Socio-Economic Index for Areas; TSI, Torres Strait Islander; UPC, Usual Provider of Care.

an empty model (no explanatory variables) using thextmelogit- command in Stata V.14. ${ }^{38}$

Models included all patient-level exposures and covariates described under those sub-headings. For the assessment of HbA1c overuse and underuse, two separate logistic regression models were used rather than an ordered logistic regression characterising underuse/ overuse/appropriate use. This was because drivers of overuse and underuse may have differed, and because there was no clear ordering of these three levels, that is, neither underuse or overuse could be considered closer to appropriate use.

\section{Effect modification}

Effect modification was assessed in relation to the number of providers and patient comorbidity variables, as these practice and patient characteristics may influence patterns of GP contact and/or the outcomes examined. Each of the four models in the main analysis (ie, one model per outcome) was repeated twice, once including interactions with the number of providers (for all three exposure variables), and once with the exposures interacted with patient comorbidity. Each of these eight unrestricted models was compared with the equivalent restricted model (ie, the main analysis for each outcome) using likelihood ratio tests using Stata's-lrtestcommand. Where the likelihood ratio test was significant, the full output of the unrestricted model was reported and interpreted.

\section{Sensitivity analysis: all diabetes}

Many of the diagnosis records mentioned diabetes without stating the type, meaning that there were likely some patients with type 2 diabetes excluded from the study cohort (as only those with a clear indication of type 2 diabetes were included). As a sensitivity analysis, we identified the cohort of all patients with diabetes (type 1, type 2 and where type was unspecified), as the outcomes assessed here are also relevant to type 1 diabetes. ${ }^{39} 40$ All analyses were repeated for this cohort to understand if this uncertainty over diabetes type may have influenced results.

\section{Patient and public involvement}

Patients were not involved in the design or conduct of this research. NPS MedicineWise maintain a web page for consumers with information on why and how their data may be used along with details of approved studies, and provide information to participating practices to display in waiting areas.

\section{RESULTS}

\section{Cohort characteristics}

Table 1 displays descriptive statistics of the 22791 individuals and 193 practices included. A flow chart detailing cohort selection is included as online supplemental file 1 . Most were male (54.2\%), lived in major cities $(63.1 \%)$, were non-Indigenous $(82.4 \%)$ and were non-smokers or ex-smokers $(82.9 \%)$, while the largest age group was $60-69(34.2 \%)$. Almost all had medications for at least one comorbidity $(96.2 \%)$. Fourteen per cent had 
Table 2 Univariate relationships between three exposures and each outcome

\begin{tabular}{|c|c|c|c|c|c|}
\hline \multirow[b]{2}{*}{ Exposure } & & \multicolumn{3}{|c|}{ Process of care outcomes } & \multirow{2}{*}{$\begin{array}{l}\text { Clinical outcome } \\
\text { HbA1c within } \\
\text { target on first test } \\
\text { in f/up }\end{array}$} \\
\hline & & HbA1c underuse & HbA1c overuse & eGFR underuse & \\
\hline \multirow[t]{3}{*}{ Regularity } & Least & 65.77 & 12.33 & 31.66 & 29.73 \\
\hline & 2 & 59.35 & 14.44 & 25.95 & 31.17 \\
\hline & Most & 58.70 & 14.61 & 26.89 & 31.28 \\
\hline Significance & & $\chi^{2}(4)=84.8, p<0.001$ & $\chi^{2}(4)=20.5, p<0.001$ & $\begin{array}{l}\chi^{2}(4)=85.2 \\
p<0.001\end{array}$ & $\chi^{2}(4)=5.4, p=0.245$ \\
\hline $\begin{array}{l}\text { Practice-specific UPC } \\
\text { index }\end{array}$ & $0-0.39$ & 56.21 & 17.65 & 24.83 & 31.51 \\
\hline Significance & & $\chi^{2}(3)=80.2, p<0.001$ & $\chi^{2}(3)=142.3, p<0.001$ & $\begin{array}{l}\chi^{2}(3)=27.3 \\
p<0.001\end{array}$ & $\chi^{2}(3)=1.9, p=0.598$ \\
\hline \multirow[t]{2}{*}{$\mathrm{CDM}^{*}$} & No & 71.54 & 9.83 & 34.54 & 29.75 \\
\hline & Yes & 53.52 & 16.82 & 22.27 & 31.36 \\
\hline Significance & & $\chi^{2}(1)=707.6, p<0.001$ & $\chi^{2}(1)=207.9, p<0.001$ & $\begin{array}{l}\chi^{2}(1)=402.5 \\
p<0.001\end{array}$ & $\chi^{2}(1)=4.9, p=0.027$ \\
\hline
\end{tabular}

For process of care outcomes $n=22791$, for the clinical outcome $n=18381$. Displaying $\%$ of each group with outcome. ${ }^{*}$ Reimbursement to GP for certain care coordination activities.

CDM, chronic disease management; eGFR, estimated glomerular filtration rate; GP, general practitioner; HbA1c, glycosylated haemoglobin; UPC, Usual Provider of Care.

records indicating HbA1c overuse during the follow-up, $60.0 \%$ underuse and $26.7 \%$ had records indicating eGFR underuse. One-quarter (24.9\%) had an HbAlc result within target on the first test during follow-up while $19.4 \%$ had no HbAlc test; of those with at least one test during follow-up the result on the first test was within target for $30.8 \%$. Most practices $(64.8 \%)$ were in major cities, $35.2 \%$ were solo practices and $8.8 \%$ had over 20 GPs. Both the regularity and UPC measures showed significant, positive correlations between the exposure and follow-up periods, indicating these measures were stable over time (online supplemental file 3 ).

Univariate analyses are presented in table 2. Informational continuity was associated with an increased likelihood of recording an $\mathrm{HbAlc}$ result within target $31.4 \%$ among those with a CDM record compared with $29.8 \%$ among those without, $\mathrm{p}=0.027$ ), though relational continuity and regularity were not. All exposures were associated with each process of care measure. Underuse of HbA1c and eGFR were each most likely among the least regular quintile and those without a CDM item, while HbAlc overuse showed the reverse. UPC results differed, HbA1c and eGFR underuse were most likely among the high relational continuity group, while $\mathrm{HbAlc}$ overuse was most likely among the low relational continuity group. There were weak correlations between each pair of exposure variables during the exposure period (online supplemental file 3 ).

\section{Model outcomes}

ICCs for each outcome are presented in table 3. The practice level explained $11.7 \%$ of the variation in $\mathrm{HbA1c}$ underuse, $15.5 \%$ of HbAlc overuse, $21.5 \%$ of eGFR underuse and $1.6 \%$ of the $\mathrm{HbAlc}$ within target range outcome.

Model outputs, displaying ORs for exposures of interest only, are presented in figure 1 with full outputs in online supplemental file 4. Higher regularity (adjusted for practice-specific UPC, CDM and other covariates) was associated with reduced odds of HbAlc underuse (OR for most regular group $0.74,95 \%$ CI 0.67 to 0.81 ) and

Table 3 Intraclass correlation coefficients (ICCs)

\begin{tabular}{ll}
\hline Outcome variable & ICC \\
\hline HbA1c underuse & 0.117 \\
\hline HbA1c overuse & 0.155 \\
\hline eGFR underuse & 0.215 \\
\hline HbA1c within target & 0.016
\end{tabular}

eGFR, estimated glomerular filtration rate; HbA1c, glycosylated haemoglobin. 
A

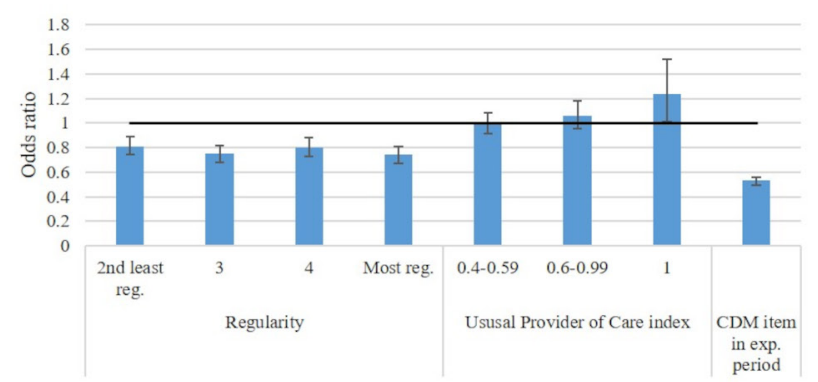

$\mathrm{C}$

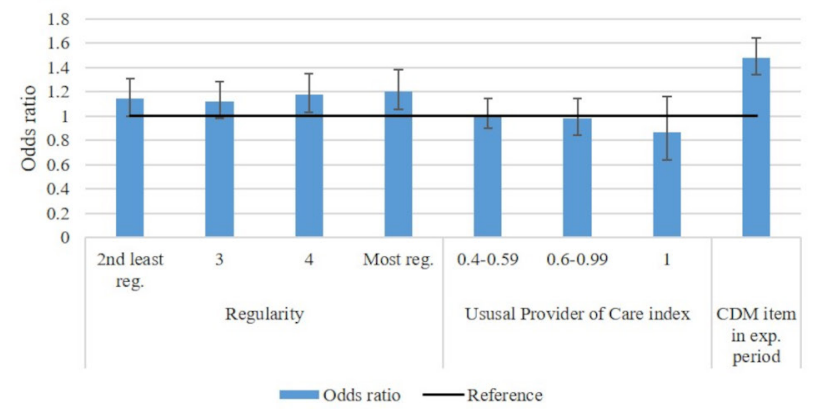

B

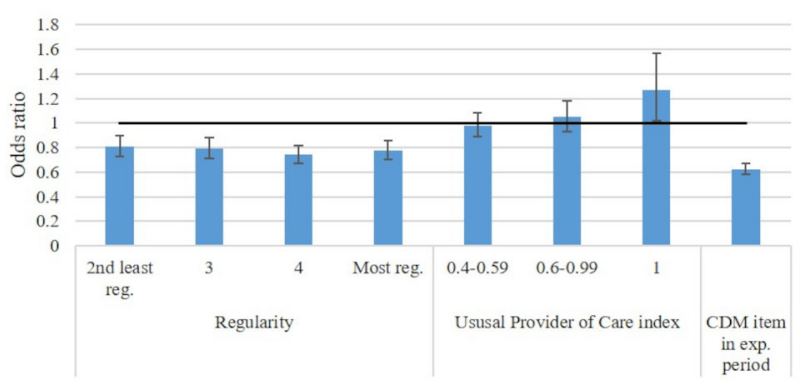

$\mathrm{D}$

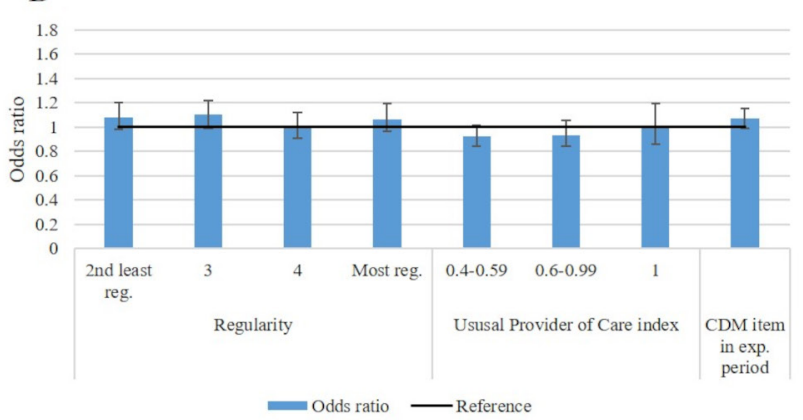

Figure 1 Outputs of regression models, ORs for exposures of main interest only. Outcomes represented are: (A) HbA1c underuse, (B) eGFR underuse, (C) HbA1c overuse and (D) recording a $\mathrm{HbA1c}$ value within target range on first measure during follow-up. Bars represent ORs while black lines represent reference values, that is, the least regular, lowest continuity and no chronic disease management ((CDM) item groups. eGFR, estimated glomerular filtration rate; HbA1c, glycosylated haemoglobin.

eGFR underuse (OR for most regular group 0.78, 95\% CI 0.70 to 0.86 ). A dose-response relationship was not observed. Higher regularity was also associated with higher odds of HbAlc overuse (OR for most regular group $1.20,95 \%$ CI 1.05 to 1.38 ). For the outcome of HbA1c result within target, ORs for regularity were positive (ie, increased odds of a result within targets) but nonsignificant. Similar patterns were seen for informational continuity, which was associated (adjusted for regularity, practice-specific UPC and other covariates) with reduced odds of HbA1c underuse (OR 0.53, 95\% CI 0.49 to 0.56 ), reduced eGFR underuse (OR $0.62,95 \%$ CI 0.58 to 0.67 ) and higher odds of HbAlc overuse (OR 1.48, 95\% CI 1.34 to 1.64$)$. This exposure also had a non-significant positive association with recording $\mathrm{HbAlc}$ within target (OR 1.07, 95\% CI 0.99 to 1.15). Results differed for the practice-specific UPC index. The perfect UPC group (adjusted for regularity, CDM and other covariates) was more likely to report HbAlc underuse (OR 1.24, 95\% CI 1.01 to 1.52 ) and eGFR underuse (OR $1.2795 \%$ CI 1.02 to 1.57 ) though no other significant associations were observed.

Of the covariates, age reported significant associations with all outcomes as did comorbidity, socioeconomic status was associated with the three process of care indicators, and indigenous status was associated with all outcomes apart from HbAlc overuse. Results for these and other covariates are available in online supplemental file 4 .

\section{Effect modification}

Outputs of likelihood ratio tests are provided in online supplemental file 3 along with full outputs of unrestricted models in cases where the likelihood ratio test was significant. The only interaction with a significant likelihood ratio test was the model of eGFR underuse with practice size as the effect modifier $\left(\chi^{2}(21)=36.55, \mathrm{p}=0.019\right)$. In this model the only significant interaction term was the interaction of having a CDM item with the largest practice size (20+ providers). The OR for this interaction was $0.71(\mathrm{p}=0.001)$ indicating that the negative association between having a CDM completed and eGFR underuse (ie, a beneficial association) was greater where the practice size was larger.

\section{Sensitivity analysis}

Outcomes of sensitivity analysis are reported in online supplemental file 5. The cohort of all people with diabetes (irrespective of type) was $34 \%$ larger than the T2DM cohort $(\mathrm{n}=30453)$. Cohort characteristics were similar, with relative sizes of groups differing by $1 \%-2 \%$.

In terms of model outcomes, the ORs for regularity did not change substantially on any outcome, with the largest being a change of 0.04 for one level of regularity in relation to HbAlc overuse, and significance mostly unchanged (the exception being one additional level of regularity being significant in relation to $\mathrm{HbAlc}$ overuse). ORs for the CDM items also matched the main analysis, with the exception of the HbAlc within target outcome for which the positive association became significant 
(likely due to the larger cohort size). Regarding practicespecific UPC, ORs for HbA1c overuse outcomes remained non-significant in all cases. Associations with HbA1c and eGFR underuse were non-significant at all levels in contrast to the T2DM cohort for which perfect UPC had a positive association with these outcomes. The moderate UPC group had a negative association with the likelihood of recording $\mathrm{HbAlc}$ within target range though the high and perfect practice-specific UPC groups had no such associations; whereas in the T2DM cohort no level of UPC was associated with this outcome.

\section{DISCUSSION}

Regularity of GP contact was associated with processes of care, as was informational continuity. Interpersonal continuity measured via practice-specific UPC was generally not associated with these outcomes, with the exception of perfect UPC (all visits to the same GP) being associated with underuse of HbA1c and eGFR. The clinical outcome, HbAlc within target, was not significantly associated with any exposure in the main analysis.

The practice level accounted for a smaller proportion of the variation in $\mathrm{HbAlc}$ results than it did for processes of care. This makes sense as the GP/practice has a direct role in test ordering while blood glucose is determined by factors over which the GP has a more indirect influence (diet) ${ }^{41}$ lifestyle, ${ }^{42}$ medication adherence, ${ }^{43}$ genetics, ${ }^{44}$ etc). This may also partially explain the lack of significant association most exposures had with HbA1c level. Similarly, GPs may decide to adopt different treatment targets for some patients. Although associations with this outcome were non-significant, ORs did consistently point in the same direction as for process of care measures, and non-significant associations may reflect a modest effect size and insufficient power for this outcome rather than clear evidence of a null effect. The fact that the CDM item exposure was associated with this outcome in the sensitivity analysis supports this.

Continuity of care was assessed using two measures with contrasting results. Informational continuity reported results in line with regularity, while relational continuity differed; perfect UPC was associated with an increased likelihood of monitoring tests being missed. The limitation of the UPC here is that patient identifiers were practice specific and not all practices participate in MedicineInsight, hence some of those with high or perfect UPC may also have visited non-participating GPs and in reality had lower continuity than observed here. Meanwhile the baseline group is made up of people with all (recorded) visits to the same practice even if different providers were seen, hence interpretation of this index differs compared with most studies. The NPS are undertaking work to allow for observation of patients across practices, ${ }^{24}$ which would allow UPC and other indices to be calculated more accurately. In $201753 \%$ of patients in Australia visited only one practice, $30 \%$ visited two, with the remainder visiting three or more. ${ }^{45}$ Studies of patient preferences have assessed whether patients value informational or relational continuity more highly, with conflicting results. ${ }^{46}{ }^{47}$ There was some evidence that the impact of CDM activities may be modified by practice size, with the benefits in terms of eGFR testing greater for larger practices, suggesting that shared care plans may become more important in settings where interpersonal continuity is more difficult to achieve. In practice relational continuity implies the presence of informational continuity, so there is never a 'trade-off' between these. Trade-offs may exist between relational continuity and access (eg, policies which promote rapid GP access may reduce the chance of seeing the same GP) ${ }^{48}$ and in this context evidence regarding the value of relational continuity in isolation from informational continuity may be meaningful.

Although this is the first work to assess associations between regular GP contacts and diabetes control, some papers have assessed the effects of continuity on comparable outcomes, with inconsistent results. One large-scale study set in Israel found that continuity (UPC) had no effect on diabetes monitoring, but was associated with lower HbA1c. ${ }^{49}$ In this study, patients could be observed visiting different practices, interpretation of UPC differed from the current work. A study from the USA using a similar continuity measure was set at a single practice, so was comparable in the measurement of relational continuity. This study reported similar findings to the work from Israel, that is, continuity had no effect on monitoring tests but led to improvements in HbAlc. ${ }^{50}$ However, another single-site study from the USA found that having a personal physician did not influence the odds of a healthy HbA1c result. ${ }^{51}$ Comparisons across settings are challenging due to differences in payment mechanisms which may influence pathology ordering, potential financial barriers faced by patients influencing visit patterns, and other contextual differences.

This study found contrasting results for HbAlc underuse and overuse outcomes. Increased regularity and informational continuity were each associated with a reduced likelihood of underuse but an increased likelihood of overuse, making interpretation challenging. Effect sizes were larger in relation to the underuse outcomes (based on absolute coefficient values), so the beneficial effects for one outcome may outweigh the negative effects on the other. Of course, this assumes that HbAlc underuse and overuse are equally problematic, which may not be the case. Nonetheless, for the eGFR test, where overuse is not an issue in the same way, beneficial associations were observed. We did not develop an overall indicator of appropriate/inappropriate testing. As the drivers of overuse and underuse appear to differ, we considered that any attempt to produce a single measure would likely obfuscate this information and provide less meaningful results. These results indicate that patterns of primary care contact may be influential in terms of the quality of care received by patients. Previous studies have suggested associations between continuity/regularity of 
care and hospitalisation outcomes, ${ }^{246}$ The current work adds to this literature by presenting some evidence for pathways via which such associations may occur, though further research may be required to understand the discrepancy in findings between process of care outcomes and the clinical indicator assessed. At the practice level, these findings reinforce the need for practices to maintain continuity with patients to support the delivery of quality care.

\section{Strengths and limitations}

This work is strong in several respects. The availability of pathology test ordering and results supported the assessment of multiple intermediate outcomes. The MedicineInsight data provided a large study cohort and important covariates at the patient and practice levels. The longitudinal design prevents reverse causation bias.

There are also limitations to this analysis. First, the lack of visit data on visits to practices not participating in MedicineInsight is a limitation, as discussed previously, which impacts the interpretation of UPC. As the data were not generated for research purposes, it is inevitable that some potentially useful information (eg, patient health behaviours and attitudes) were mostly unavailable (except smoking status). This work aims to better understand causal pathways influencing hospitalisation outcomes. Being a general practice collection, MedicineInsight does not include information on hospitalisations, hence it was not possible to investigate the role of these intermediate outcomes as mediators.

Results reported here may differ outside of the Australian context. Different registration systems, financial barriers, payment systems and diagnostic test ordering approaches must be considered when considering relationships elsewhere. Although findings of the sensitivity analysis capturing all people with diabetes were generally similar to the main analysis of T2DM, there were some minor differences in results. This may suggest that findings here may not be generalisable to cohorts with type 1 diabetes.

Missing data can be an issue in any study making secondary use of routinely collected data. We have managed this issue by using the fields least likely to suffer from missing data for our exposures and outcomes of main interest. Our exposures, being patterns of GP contact, only rely on a record being created for each GP visit, while the pathology data used for outcomes are transmitted electronically from pathology labs to practice CIS's and results stored in the correct fields. Missing information on covariates was managed by recoding these records as a separate category to prevent data loss.

Finally, in interpreting the outcome of HbAlc results, patients with no HbAlc tests during follow-up were necessarily omitted, which could bias findings. If lower regularity is associated with both a reduced likelihood of HbA1c testing being performed (which these results suggest), and poorer HbA1c control, the omission of those patients with no tests during follow-up would bias results towards a null or negative effect.

\section{CONCLUSION}

Previous works have demonstrated associations between regularity/continuity of GP contact and hospitalisation outcomes, with authors hypothesising that any beneficial effects result from improved patient monitoring and treatment. This analysis demonstrated that among patients with T2DM, more regular GP contact was associated with a reduced likelihood of monitoring tests being missed, but this is balanced against an increased likelihood of overtesting, which may represent inefficient use of resources and potentially suboptimal patient care. Overall regular GP contacts were associated with small, non-significant associations with the likelihood of recording HbA1c results within a healthy range. Similar results were observed in relation to informational continuity. It is plausible that associations with hospital and emergency department outcomes occur via these intermediate outcomes.

\section{Twitter Suzanne Robinson @Robinsonsuz}

Acknowledgements The authors wish to acknowledge the NPS for provision of data, and the practices and patients whose data contributed to this study. We also thank Cameron Wright for his advice.

Contributors DY designed the study, performed analyses, wrote the first version of the manuscript and is the guarantor. RM and JD contributed to study design, analyses and choice of outcomes from clinical guidelines. MNH provided advice on statistical issues. SR advised on analyses and interpretation of findings. All authors reviewed manuscript drafts and approved the final version.

Funding This work was supported by the National Health and Medical Research Council (APP 1078345) and a PhD scholarship via the Australian Government Research Training Programme.

Competing interests RM and MNH report grants from the National Health and Medical Research Council (Australia) during the conduct of the study. DY reports grants from the Research Training Programme (Australia) during the current study.

Patient consent for publication Not applicable.

Ethics approval Approvals were granted by the Curtin University Human Research Ethics Committee, HRE2017-0579, and the Medicinelnsight independent external Data Governance Committee on 22 August 2017, reference 012-2017.

Provenance and peer review Not commissioned; externally peer reviewed.

Data availability statement Data may be obtained from a third party and are not publicly available. Data supporting these findings can be requested from the custodians of the relevant data collection. Restrictions placed by the data custodians mean that data used for this study cannot be made publicly available.

Supplemental material This content has been supplied by the author(s). It has not been vetted by BMJ Publishing Group Limited (BMJ) and may not have been peer-reviewed. Any opinions or recommendations discussed are solely those of the author(s) and are not endorsed by BMJ. BMJ disclaims all liability and responsibility arising from any reliance placed on the content. Where the content includes any translated material, BMJ does not warrant the accuracy and reliability of the translations (including but not limited to local regulations, clinical guidelines, terminology, drug names and drug dosages), and is not responsible for any error and/or omissions arising from translation and adaptation or otherwise.

Open access This is an open access article distributed in accordance with the Creative Commons Attribution Non Commercial (CC BY-NC 4.0) license, which permits others to distribute, remix, adapt, build upon this work non-commercially, and license their derivative works on different terms, provided the original work is properly cited, appropriate credit is given, any changes made indicated, and the use is non-commercial. See: http://creativecommons.org/licenses/by-nc/4.0/. 


\section{ORCID iDs}

David Youens http://orcid.org/0000-0002-4296-4161

Suzanne Robinson http://orcid.org/0000-0001-5703-6475

Rachael Moorin http://orcid.org/0000-0001-8742-7151

\section{REFERENCES}

1 Haggerty JL, Reid RJ, Freeman GK, et al. Continuity of care: a multidisciplinary review. BMJ 2003;327:1219-21.

2 Moorin RE, Youens D, Preen DB, et al. Association between continuity of provider-adjusted regularity of general practitioner contact and unplanned diabetes-related hospitalisation: a data linkage study in New South Wales, Australia, using the 45 and Up Study cohort. BMJ Open 2019;9:e027158.

3 Youens D, Harris M, Robinson S, et al. Regularity of contact with GPs: measurement approaches to improve valid associations with hospitalization. Fam Pract 2019;36:650-6.

4 Chen C, Cheng S. Better continuity of care reduces costs for diabetic patients. American Journal of Managed Care 2019;6:420-7.

5 Comino EJ, Islam MF, Tran DT, et al. Association of processes of primary care and hospitalisation for people with diabetes: a record linkage study. Diabetes Res Clin Pract 2015;108:296-305.

6 Hong J-S, Kang H-C. Continuity of ambulatory care and health outcomes in adult patients with type 2 diabetes in Korea. Health Policy 2013;109:158-65

7 Katz DA, McCoy KD, Vaughan-Sarrazin MS. Does greater continuity of Veterans administration primary care reduce emergency department visits and hospitalization in older veterans? J Am Geriatr Soc 2015;63:2510-8.

8 Gill JM, Mainous AG. The role of provider continuity in preventing hospitalizations. Arch Fam Med 1998;7:352-7.

9 Romaire MA, Haber SG, Wensky SG, et al. Primary care and specialty providers: an assessment of continuity of care, utilization, and expenditures. Med Care 2014;52:1042-9.

10 Ride J, Kasteridis P, Gutacker N, et al. Impact of family practice continuity of care on unplanned Hospital use for people with serious mental illness. Health Serv Res 2019;54:1316-25.

11 Mainz J. Defining and classifying clinical indicators for quality improvement. Int J Qual Health Care 2003;15:523-30.

12 Youens D, Doust J, Robinson S. Regularity and continuity of GP contacts and use of statins among people at risk of cardiovascular events. Journal of General Internal Medicine 2020.

13 Duckett S, Wilcox S. The Australian health care system. 5th ed. Melbourne: Oxford University Press, 2015.

14 Peabody JW, Bickel SR, Lawson JS. The Australian health care system. are the incentives down under right side up? JAMA 1996;276:1944-50.

15 Keleher H. The Medical Profession in Australia. In: Mcintyre T, ed. Understanding the Australian health care system. 3rd ed. NSW: Elsevier, 2016.

16 Sarrazin MSV, Rosenthal GE. Finding pure and simple truths with administrative data. JAMA 2012;307:1433-5.

17 Youens D, Moorin R, Harrison A, et al. Using general practice clinical information system data for research: the case in Australia. Int $J$ Popul Data Sci 2020;5:1099.

18 Australian Institute of Health and Welfare. Diabetes. Canberra Australian Government; 2020. https://www.aihw.gov.au/reports/ australias-health/diabetes [Accessed cited 09 Sep 2020].

19 Royal Australian College of General Practitioners. General practice management of type 2 diabetes: 2016-18 East Melbourne, Vlc; 2016

20 Shrivastav M, Gibson W, Shrivastav R, et al. Type 2 diabetes management in primary care: the role of retrospective, professiona continuous glucose monitoring. Diabetes Spectr 2018;31:279-87.

21 Lee CMY, Mnatzaganian G, Woodward M, et al. Sex disparities in the management of coronary heart disease in general practices in Australia. Heart 2019;105:1898-904.

22 Mnatzaganian G, Lee CMY, Robinson S, et al. Socioeconomic disparities in the management of coronary heart disease in 438 general practices in Australia. Eur J Prev Cardiol 2020:400-7.

23 Benchimol El, Smeeth L, Guttmann A, et al. The reporting of studies conducted using observational Routinely-collected health data (RECORD) statement. PLoS Med 2015;12:e1001885.

24 Busingye D, Gianacas C, Pollack A, et al. Data resource profile: Medicinelnsight, an Australian National primary health care database. Int J Epidemiol 2019;48:1741-1741h

25 NPS MedicineWise. Medicinelnsight data book version 2.1. Sydney: NPS MedicineWise, 2018.
26 NPS MedicineWise. Medicinelnsight, 2021. Available: https://www. nps.org.au/medicine-insight

27 NPS MedicineWise. Using Medicinelnsight data for research, 2018. Available: https://www.nps.org.au/medicine-insight/usingmedicineinsight-data

28 Yakaryılmaz FD, Öztürk ZA. Treatment of type 2 diabetes mellitus in the elderly. World J Diabetes 2017;8:278-85.

29 Department of Health. Medicare benefits schedule book. Canberra: Australian Government, 2019.

30 Rodriguez HP, Marshall RE, Rogers WH, et al. Primary care physician visit continuity: a comparison of patient-reported and administratively derived measures. J Gen Intern Med 2008;23:1499-502.

31 Sherwani SI, Khan HA, Ekhzaimy A, et al. Significance of HbA1c test in diagnosis and prognosis of diabetic patients. Biomark Insights 2016;11:95-104.

32 McCoy RG, Van Houten HK, Ross JS, et al. HbA1c overtesting and overtreatment among US adults with controlled type 2 diabetes, 2001-13: observational population based study. BMJ 2015;351:h6138.

33 Australian Bureau of Statistics. The Australian statistical geography standard (ASGS) remoteness structure, 2020. Available: https:// www.abs.gov.au/websitedbs/d3310114.nsf/home/remoteness+ structure

34 Pratt NL, Kerr M, Barratt JD, et al. The validity of the Rx-Risk comorbidity index using medicines mapped to the anatomical therapeutic chemical (ATC) classification system. BMJ Open 2018;8:e021122.

35 Australian Bureau of Statistics. Socio-Economic indexes for areas, 2018. Available: https://www.abs.gov.au/websitedbs/censushome. nsf/home/seifa

36 Cowling TE, Harris M, Majeed A. Extended opening hours and patient experience of general practice in England: multilevel regression analysis of a national patient survey. BMJ Qual Saf 2017;26:360-71.

37 Howard M, Brazil K, Akhtar-Danesh N, et al. Self-Reported teamwork in family health team practices in Ontario: organizational and cultural predictors of team climate. Can Fam Physician 2011;57:e185-91.

38 StataCorp. Stata. College Station, Texas StataCorp; 2015.

39 Diabetes Australia. Blood glucose monitoring, 2020. Available: https://www.diabetesaustralia.com.au/living-with-diabetes/ managing-your-diabetes/blood-glucose-monitoring/

40 Diabetes australia. bladder \& Kidneys, 2020. Available: https:// www.diabetesaustralia.com.au/living-with-diabetes/preventingcomplications/bladder-kidneys/

41 Batis C, Mendez MA, Sotres-Alvarez D, et al. Dietary pattern trajectories during 15 years of follow-up and $\mathrm{HbA} 1 \mathrm{c}$, insulin resistance and diabetes prevalence among Chinese adults. $J$ Epidemiol Community Health 2014;68:773-9.

42 Kriska A, El Ghormli L, Copeland KC, et al. Impact of lifestyle behavior change on glycemic control in youth with type 2 diabetes. Pediatr Diabetes 2018;19:36-44.

43 Farmer AJ, Rodgers LR, Lonergan M, et al. Adherence to oral glucose-lowering therapies and associations with 1-year $\mathrm{HbA1c}$ : a retrospective cohort analysis in a large primary care database. Diabetes Care 2016;39:258-63

44 Leong A, Wheeler E. Genetics of HbA1c: a case study in clinical translation. Curr Opin Genet Dev 2018;50:79-85.

45 NPS Medicinewise. General practice insights report July 2016-June 2017: a working paper. Sydney NPS Medicinewise; 2018.

46 Turner D, Tarrant C, Windridge K, et al. Do patients value continuity of care in general practice? An investigation using stated preference discrete choice experiments. J Health Serv Res Policy 2007;12:132-7

47 Hudson BF, Best S, Stone P, et al. Impact of informational and relational continuity for people with palliative care needs: a mixed methods rapid review. BMJ Open 2019;9:e027323.

48 Oliver D, Deal K, Howard M, et al. Patient trade-offs between continuity and access in primary care interprofessional teaching clinics in Canada: a cross-sectional survey using discrete choice experiment. BMJ Open 2019;9:e023578.

49 Lustman A, Comaneshter D, Vinker S. Interpersonal continuity of care and type two diabetes. Prim Care Diabetes 2016;10:165-70.

50 Younge R, Jani B, Rosenthal D, et al. Does continuity of care have an effect on diabetes quality measures in a teaching practice in an urban underserved community? J Health Care Poor Underserved 2012;23:1558-65.

51 Hueston WJ. Does having a personal physician improve quality of care in diabetes? J Am Board Fam Med 2010;23:82-7. 